\title{
Attempted suicide v. non-suicidal self-injury: behaviour, syndrome or diagnosis? ${ }^{\dagger}$
}

Aine M. Butler and Kevin Malone

\section{Summary}

Nomenclature used to describe acts of self-harm without fatal consequences varies considerably. The term 'nonsuicidal self-injury' (NSSI) offers an opportunity to clarify study in this field, dichotomising the presence or absence of suicidal intent. This may improve consistency in reporting suicide and self-injurious behaviour with implications for management and prognosis.

\section{Declaration of interest}

None.
Dr Aine M. Butler is a senior registrar at St John of God Hospital and a special lecturer in psychiatry at University College Dublin and St Vincent's University Hospital. Kevin Malone (pictured) is Professor of Psychiatry at the School of Medicine and Medical Science at University College Dublin and St Vincent's University Hospital.

Non-suicidal self-injury (NSSI) has been proposed as a new diagnostic category within DSM-5. ${ }^{1}$ The study of suicide and self-injurious behaviour has been plagued by inconsistent terminology over the years. The concept of self-injury arose from Kreitman's term 'parasuicide' used to label all non-accidental, selfpoisoning or self-injury that did not result in death, regardless of the intention of the act. ${ }^{2}$ Over time the term 'deliberate self-harm' replaced parasuicide, however more recently the word 'deliberate' has been dropped because of its pejorative connotations and lack of favour with patients. ${ }^{2}$ Within the USA 'attempted suicide' has been the more commonly used term to refer to acts of self-injury with or without suicidal intent. ${ }^{3}$ Confusingly, within the USA the phrase 'deliberate self-harm' often refers to repetitive superficial bodily harm without suicidal intent, more frequently termed 'self-injury' in the UK. ${ }^{3}$

This varied nomenclature covers a spectrum of behaviour from suicide to suicide attempt to acts of self-injury without suicide intent. ${ }^{3}$ Although some believe these various forms of self-injurious behaviour merely represent different degrees of lethality of suicide attempt, there is a growing body of evidence that the motives for NSSI are different. ${ }^{4}$ If NSSI is distinct in intent, function and epidemiology, then separating it from suicide attempt may be important. Many fundamental aspects of NSSI remain unknown, and the lack of clear separation and diagnostic clarity between suicide attempt and NSSI is likely to have hampered the development of a robust evidence base for NSSI, with an impact on the development of treatment for this phenomenon.

\section{Definition of NSSI}

Non-suicidal self-injury is the direct, deliberate destruction of one's own body tissue in the absence of intent to die. It differs from suicide attempt with respect to intent, lethality, chronicity, methods, cognitions, reactions, aftermath, demographics and prevalence. Primarily, the person's intent in NSSI is not to terminate consciousness but to modify it. Common forms of NSSI include cutting, burning, scratching, banging, hitting, biting and

†See pp. 326-328, this issue. excessive rubbing. Preceding the act of self-injury is a psychological experience of increasing anger, tension, anxiety, dysphoria and general distress or depersonalisation, which the person feels they cannot escape from or control. Engaging in NSSI provides a temporary release from these distressing emotions. Other reasons include: self-punishment; to draw attention so that other people can see their distress; to make other people feel guilty and change their behaviour; and to tie in socially with peers who self-injure. ${ }^{5}$ An immediate feeling of relief, gratification and/or release from depersonalisation follows the act of self-injury. However, despite this transient response, NSSI leads to longer-term negative consequences. Emotionally, it can evoke abiding complex feelings of guilt and shame towards the self.

\section{Prevalence of NSSI and associated factors}

The difference in nomenclature and the lack of appropriately standardised and validated assessment tools for NSSI limits our understanding of its true incidence and prevalence. The majority of work evaluating NSSI has been on adolescents and young adults as a result of the belief that it is a phenomenon that declines across the course of a lifetime. One study has attempted to compare prevalence rates of NSSI in young adults across nations using comparable groups and study design. ${ }^{6}$ They compared NSSI, suicide attempts, suicide threats and suicidal ideation in a similar age group of adolescents, 14- to 17-year-olds, using the same validated assessment tools in both Germany and the USA. Findings revealed similar rates of adolescent engagement in at least one act of NSSI, with a prevalence of $25.6 \%$ in German adolescents compared with $23.6 \%$ in the US participants. Additional studies that distinguish between self-injury and suicide attempt in this way will allow clarity on prevalence rates across cultures.

Current research findings point towards diagnostic heterogeneity in those engaging in NSSI. Originally it was viewed as a symptom of borderline personality disorder; however, we now know that NSSI has been identified in numerous clinical samples without borderline personality disorder, including those with mood and anxiety disorders, eating disorders, substance misuse, conduct disorder and post-traumatic stress disorder. ${ }^{7}$ In the context of established NSSI, the extent of self-injury engaged in may play an important role in the prediction of more serious outcomes. Individuals who engage in moderate to severe self-injury are more likely to report a history of psychiatric treatment, admission to hospital, suicide attempt and suicidal ideation. ${ }^{7}$ Clarifying the extent of NSSI engaged in may be beneficial in assisting health professionals assess the level of risk associated with such behaviours. 


\section{NSSI and suicidality}

The importance of identifying NSSI lies not only in its associated negative emotional, physical and social consequences but also in its association with suicide attempts. It has been found that NSSI is the strongest predictor of future suicide attempts in adolescents with depression receiving treatment under randomised controlled trial conditions. ${ }^{8}$ High levels of depression, suicidal ideation and hopelessness characterise participants who engage in either NSSI or suicide attempt. ${ }^{8}$ These findings suggest that there are common risk factors predisposing to both NSSI and suicide attempt. Nonetheless, clear differences have been found in the extent and quality of these common clinical factors, which supports the hypothesis that NSSI is a distinct entity in its own right. Those who attempt suicide when compared with those who engage in NSSI have higher scores on anxiety, depression and suicide ideation measurements. ${ }^{8}$

One theory explaining the association is based on the hypothesis that engaging in NSSI predisposes to suicidal attempt. ${ }^{9}$ Potentially traumatic experiences such as NSSI, child abuse and combat exposure may desensitise an individual to pain and fear of self-destruction, so making suicide attempt more likely. ${ }^{9} \mathrm{~A}$ further possibility is that with a lower combination of risk factors only NSSI may be apparent. However, with a greater loading of psychopathology and environmental difficulties, one sees more frequent and persistent NSSI and eventually suicide attempt. This would suggest that although NSSI may not directly lead to suicide attempt, if the needs and deficits reflected in NSSI are not addressed it may ensue.

Notwithstanding the important association between NSSI and suicide attempt or the hypothesis that it lies along a spectrum of self-destructive actions, it is essential to remember that NSSI does not necessarily represent a less lethal attempt at suicide. The clear distinction is that whereas NSSI represents a maladaptive coping mechanism to regulate overwhelming emotions and to endure life, a suicide attempt reflects a desire to escape and to end one's life. Non-suicidal self-injury can be understood as a signal of psychological distress, which could increase risk for suicide. ${ }^{1}$ Non-suicidal self-injury and suicide attempt can occur in isolation yet they may also co-occur, there may even be alternation between NSSI and suicide attempt in the same individual. ${ }^{10}$ Yet, this does not mean that NSSI and suicide attempt are the same; essential qualitative and phenomenological differences do distinguish suicide attempt from NSSI so continuing to differentiate selfinjury with and without suicidal intent is essential to building precise understandings of these behaviours. ${ }^{10}$

\section{NSSI as a separate category in DSM-5}

Given the lack of intent to die we believe to be inherent in NSSI it is certainly feasible to separate suicide attempt from NSSI. At present, there is nowhere in the current classification of Axis I disorders to record NSSI, implying that mental health professionals need not consider it abnormal. Given the intense feelings of psychological distress, the adverse consequences associated with NSSI and its potential relationship with future suicide attempts, the importance of classifying it along with other mental health disorders is clear. At present the occurrence of NSSI may lead clinicians to make an automatic assumption that there is an underlying diagnosis of borderline personality disorder. Yet, the individual in question may actually meet diagnostic criteria for another mental health disorder. Assigning a specific category to NSSI in DSM-5 allows for improved diagnostic clarity. This may reduce the opportunity for clinician bias that NSSI merely indexes borderline personality disorder. Furthermore, NSSI may have important prognostic implications. That NSSI is likely to be a predictor of suicide attempt, as detailed earlier, adds to the evidence that it is clinically important and warrants identification. A further factor to consider is treatment of NSSI. The short- and long-term adverse consequences of NSSI suggest that treatment needs to be considered in its own right. Assigning NSSI as a separate disorder is likely to increase the chance that specific treatment will be offered. Research to date on specific treatment options for NSSI has been hampered by the lack of precision in its definition and measurement. Adding NSSI as a separate category in the new DSM-5 classification system will hopefully enhance our ability to build an evidence base for treatment options.

\section{Future directions for NSSI research}

There is mounting evidence that NSSI and suicide attempt appear to be separate entities. The proposed classification of NSSI as a separate category in DSM-5 will allow the development and validation of adequate assessment tools specific to this behaviour. Accurately distinguishing NSSI from suicide attempt will provide more information on its prevalence and allow a correct assessment of both public health implications and treatment needs. Further research using the clarified diagnostic criteria could then delineate the true aetiological, motivating and maintaining factors associated with NSSI and its real prognostic implications. This will inform the design of specific treatments and facilitate methodologically sound treatment studies testing specific interventions for NSSI in the presence and absence of other psychopathologies.

Aine M. Butler, PhD, MRCPsych, St John of God Hospital, St Vincent's University Hospital and Univeristy College Dublin; Kevin Malone, MD, FRCPI, FRCPsych, Department of Psychiatry and Mental Health Research, School of Medicine and Medical Science at University College Dublin and St Vincent's University Hospital, Dublin, Ireland

Correspondence: Aine M. Butler, St John of God Hospital, Stillorgan, County Dublin, Ireland. Email: aine.butler@sjog.ie

First received 17 Apr 2012, final revision 24 Oct 2012, accepted 31 Oct 2012

\section{References}

1 American Psychiatric Association. Non-suicidal self injury. In American Psychiatric Association DSM-5 Development. APA, 2012 (http://www.dsm5.org)

2 O'Connor R, Platt S, Gordon J. International Handbook of Suicide Prevention. Research, Policy and Practice. Wiley-Blackwell, 2011.

3 Skegg K. Self-harm. Lancet 2005; 366: 1471-83.

4 Nock MK, Kessler RC. Prevalence of and risk factors for suicide attempts versus suicide gestures: analysis of the National Comorbidity Survey. J Abnorm Psychol 2006; 115: 616-23.

5 Wilkinson P, Goodyer I. Non-suicidal self-injury. Eur Child Adolesc Psychiatry 2011; 20: 103-8.

6 Plener PL, Libal G, Keller F, Ferget JM, Muehlenkamp JJ. An international comparison of adolescent non-suicidal self-injury (NSSI) and suicide attempts: Germany and the USA. Psychol Med 2009; 39: 1549-58.

7 Jacobson CM, Muehlenkamp JJ, Miller AL, Turner JB. Psychiatric impairment among adolescents engaging in different types of deliberate self-harm. J Clin Child Adolesc Psychol 2008; 37: 363-75.

8 Wilkinson P, Kelvin R, Roberts C, Dubicka B, Goodyer I. Clinical and psychosocial predictors of suicide attempts and nonsuicidal self-injury in the Adolescent Depression Antidepressants and Psychotherapy Trial (ADAPT). Am J Psychiatry 2011; 168: 495-501.

9 Brent D. Nonsuicidal self-injury as a predictor of suicidal behavior in depressed adolescents. Am J Psychiatry 2011; 168: 452-4.

10 Muehlenkamp JJ, Claes L, Havertape L, Plener PL. International prevalence of adolescent non-suicidal self-injury and deliberate self-harm. Child Adolesc Psychiatry Ment Health 2012; 6: 10. 